\title{
Metastatic Primary Adenocarcinoma of Colon: A Case Report of 15-Year-Old Boy
}

\author{
Noemi Maria Giovanna Ognibene ${ }^{1 *}$, Massimo Basile ${ }^{2}$, \\ Raffaele Schiavone ${ }^{2}$, Marco Di Maurizio², Claudio Defilippi ${ }^{2}$ \\ ${ }^{1}$ Radiodiagnostic and Oncological Radiotherapy Unit, University Hospital “Policlinico- Vittorio Emanuele", Catania, Italy \\ ${ }^{2}$ Department of Radiology, Pediatric Radiology, Meyer Children's University Hospital, Florence, Italy
}

Received: March 17, 2016; Accepted: April 22, 2016; Published: April 28, 2016

*Corresponding author: Noemi Maria Giovanna Ognibene MD; Radiodiagnostic and Radiotherapy Unit, University Hospital "Policlinico-Vittorio Emanuele”, Via Santa Sofia 78, 95123, Catania, Italy; Tel: +393284724510, E-mail address: noeminox@hotmail.comv

\begin{abstract}
Colorectal carcinomas are rare disease in the pediatric population, especially without known risk factors. The present article reports the case of a 15 year old boy with sporadic colon carcinoma who presented with acute intestinal obstruction symptoms during a soccer match. An annular colonic mass proximal to the hepatic flexure causing mechanical obstruction was observed on abdominal enhanced magnetic resonance. The diagnosis was confirmed by emergency laparotomy which identified an infiltrative and stenosing colonic mass, with serosal involvement and hepatic metastasis. Histological examination of the mass revealed a moderately differentiated adenocarcinoma. The present study aimed to suggest that malignancies should be considered by pediatricians and pediาatric surgeons for differential diagnosis when patients present with intestinal obstruction symptoms such as unexplained persistent constipation, abdominal distension or bleeding per rectum, as early diagnosis and treatment decreases the mortality rate of patients.
\end{abstract}

Keywords: Colon Adenocarcinoma; Childhood Tumors; Intestinal Obstruction; Liver Metastasis

\section{Abbreviations}

CRC: Colorectal Carcinoma; CT: Computed Tomography, DNA: Deoxyribonucleic Acid, FAP: familial Adenomatous Polyposis, HNPCC: Hereditary Nonpolyposis Colorectal Cancer Syndrome, MR: Magnetic Resonance, MSI: Microsatellite Instability, pTMN: Pathologic classification of colorectal cancer staging system by American Joint Committee on Cancer (AJCC), primary Tumor (T),status of the regional Nodes (N), and distant Metastasis (M).

\section{Introduction}

Colorectal cancer (CRC) is a rare pediatric tumor, representing only $1 \%$ of all pediatric malignancies, with an incidence rate of between 1 and 8 cases per million children worldwide [1-3]. It is also the most common primary gastrointestinal malignancy in children [4,5].Childhood CRCs more frequently develops in children without known predisposing syndromes, nonetheless, $10 \%$ to $30 \%$ of it have predisposing factors [4] which include familial adenomatous polyposis (FAP), ulcerative colitis, Crohn disease and Peutz-Jegher syndrome [6]. High microsatellite instability (MSI),caused by a DNA mismatch repair system, is one of the most important genetic mutations in childhood CRC [6] and is the hallmark of the early-onset CRC, Lynch syndrome, which is also known as hereditary nonpolyposis colorectal cancer syndrome (HNPCC).

Alot of studies demonstrated differences between pediatric and adults CRC patients, showing a significantly higher proportion of aggressive histology, particularly signet ring and mucinous and a higher proportion presenting with metastatic disease [7-11]. It is not clear why children more commonly present at a later stage than adults, but postulated causes include delays in diagnosis owing to its low incidence and mimicry of more common pathologies including infectious and inflammatory bowel disease [12]. The majority of cases of pediatric colorectal carcinoma are identified in older children: $70-95 \%$ of cases occur in children $>10$ years of age [1, 3, 13-15]. Childhood colon cancers have a poor prognosis, with a reported 5-year survival ranging from 7 to $12 \%$ [1, 3, 14-17]. This poor prognosis may be related to a delayed diagnosis, to the higher incidence of malignant histology, and to the potential of a more rapid tumor growth in a subject who is actively growing [18]. Contrast studies, ultrasonography and computed tomography are essential procedures for determining the extent of the disease. Postoperative histological examination is considered the gold standard for diagnosis.

CRC, although rare, should be suspected and investigated in any child presenting with unexplained persistent constipation, abdominal distension or bleeding per rectum [19]. The present study reports the case of colonic carcinoma in a 15-year-old male patient without any risk factors. We also review the clinical, pathological and imaging characteristics of young-age sporadic colorectal cancer (CRC).

\section{Case Report}

A 15-year-old male patient without any previous medical history presented in emergency department with primary complaints of several abdominal pain during a soccer match. Family history revealed nothing of significance. An abdominal X-ray no revealed air-fluid levels. He had an abdominal ultrasound examination which showed multiple hepatic lesions suspected for metastasis. Abdominal enhanced computed tomography (CT) scan was then immediately performed which showed multiple and coarse focal liver lesions with typical "target appearance" compatible with secondary injury, multiple and increased mesenteric 
lymph nodes gathered in packets and bowel wall thickening proximal the hepatic flexure.

Transferred in our hospital an abdominal enhanced magnetic resonance (MR) was perfomed confirming the presence of multiple nodular secondary lesions distributed in all hepatic segments (figure1E), significant stenosis in transverse colon near to the hepatic flexure, with local bowel wall thickening (figure 1, 2, 3); therefore, an occupying lesion could not be excluded; enlarged and confluent mesenteric lymph nodes (figure 4) and significant distention with air-fluid levels of the ascending colon and ileum. Following 3 days of conservative treatment, an abdomen X-ray (figure 5) revealed an obstruction of the upper section of the colon with air-fluid levels and an emergency laparotomy was performed in order to relieve the intestinal obstruction: a mass measuring $5 \times 4 \mathrm{~cm}$ was identified in the transverse colon, which formed a near complete obstruction of the enteric cavity. The transverse colon where the mass was located was excised ( $5 \mathrm{~cm}$ surgical margin around the tumor) and a colo-colonic anastomosis was performed. Following proximate bowel decompression, dissection of retroperitoneal lymphadenopathy was performed and liver were explored, numerosous hepatic metastasis were confirmed.

Histological examination of the mass revealed moderately differentiated adenocarcinoma Lymphovascular invasion was positive. Tumor metastasized to the liver and the lymph nodes. The proximal and distal resection margins were free. Pathologic stage was IVA (pT4aN1aM1a).

For HNPCC evaluation, immunohistochemical staining for MLH1 and MSH2 was done, which gave a normal result. RT-PCR analysis did not detect microinstability in any of the markers tested.
FDG-PET demonstrated no other metastases.

He is performing postoperative adjuvant chemotherapy and hepatic metastasis radio-embolisation (figure 6, 7).

After 10 months of follow-up, no tumor local recurrence was noted.

\section{Discussions}

Primary gastrointestinal system malignancies constitute $2 \%$ of pediatric neoplasms and colorectal cancer is the second most common malignancy after primary tumor of the liver $[19,21]$. CRC is rare in children, with annual incidence of about one in 10 million adolescents younger than 20 years old [22]. The reported peak age is 15 years old, as our patient, while the youngest reported patient is a 9-month-old female infant [5]. In contrast to the pediatric population, CRC is generally considered to be a disease of older persons; more than $90 \%$ of colorectal cancer patients is above 55 years old [23]; it is the third most common cancer in men and the second in women [24]. Pediatric CRC may arise in the setting of predisposing genetic or polyposis syndromes such as FAP, Lynch syndrome or familial juvenile polyposis, as well as in the setting of inflammatory bowel disease, but CRC more frequently develops in children without known predisposing syndromes [25]. We reported this case as a sporadic CRC because the patient had neither family history of colon cancer nor any other malignancy.

In adulthood, the most frequent colorectal cancers are moderately differentiated or well-differentiated adenocarcinomas, while the poorly differentiated to undifferentiated, mucinous type, signet-ring cellcontaining carcinomas is higher in younger patients. Not only have they a poorer response to chemotherapy, but also associated with extensive intramural spread and peritoneal carcinomatosis [4]. Kravarusic et al.
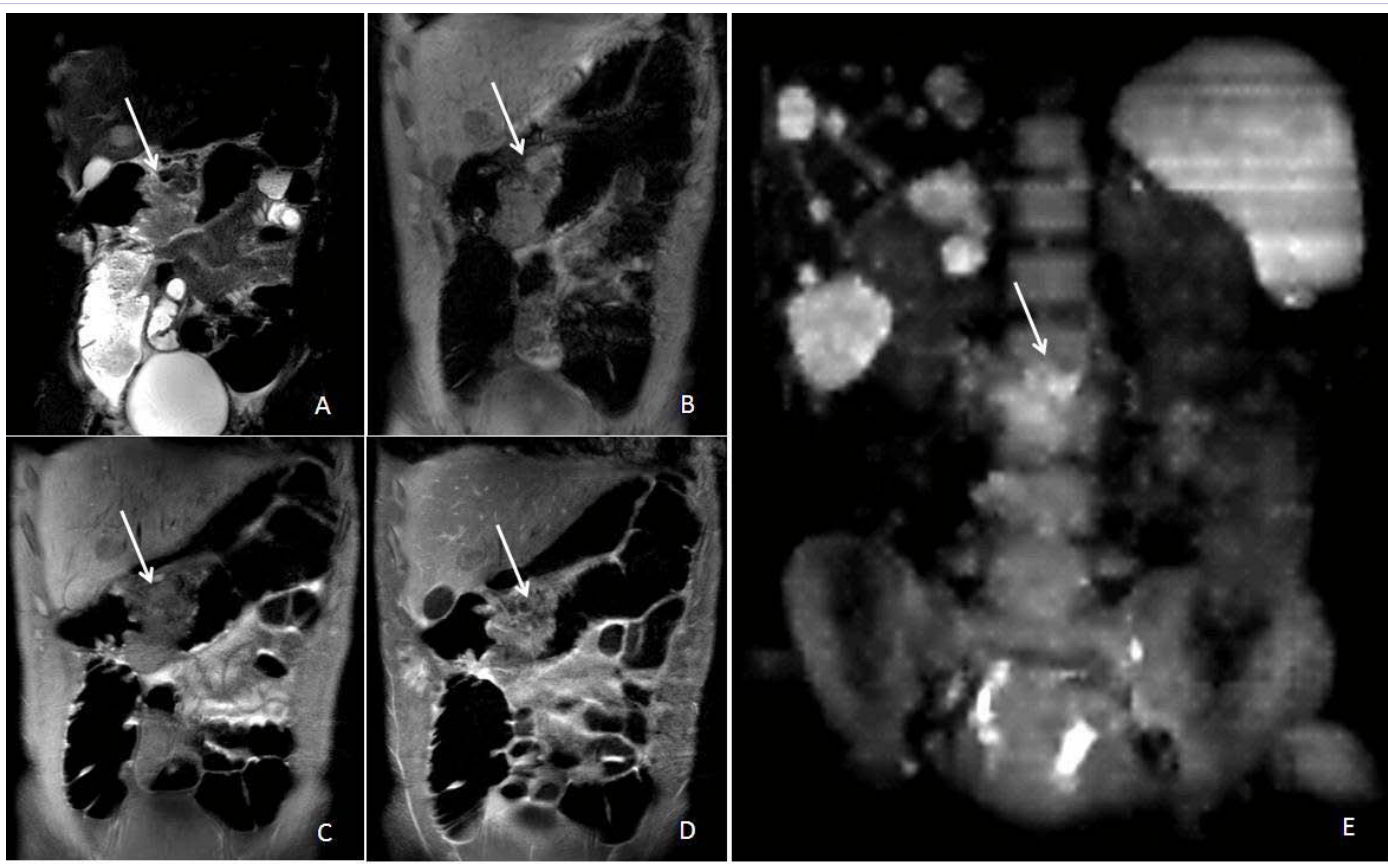

Figure 1: Coronal fat-suppressed T2-weighted (A), DIXON (B), unenhanced (C) and enhanced (D) fat-suppressed T1-weighted MR images show a significant stenosis in transverse colon near to the hepatic flexure, with local bowel wall thickening (white arrow). 3D-DWIBS MR reconstruction (b = $1000 \mathrm{~s} / \mathrm{mm}^{2}$ ) (E) shows that the bowel wall thickening (white arrow) and multiple hepatic lesion demonstrate restricted diffusion. 

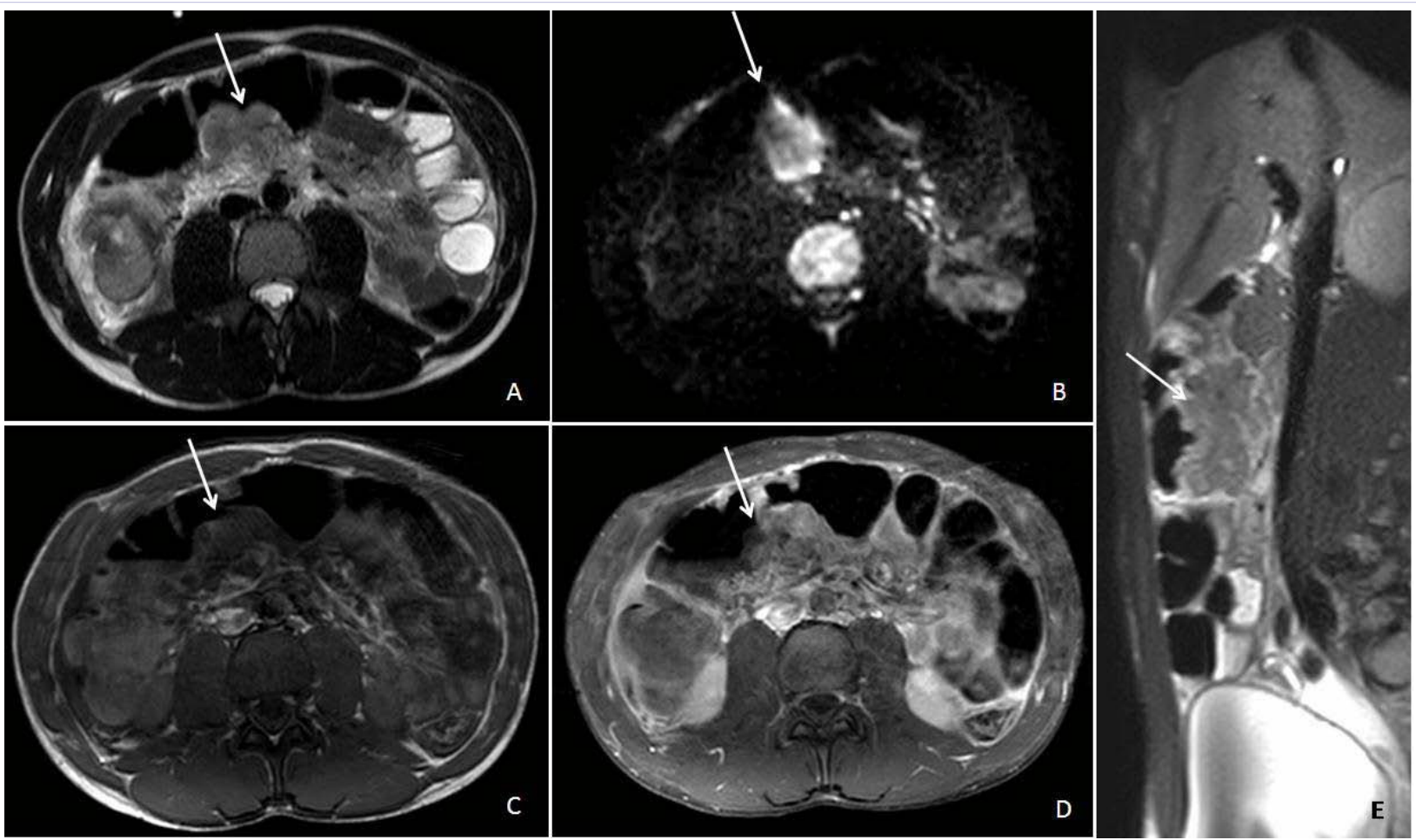

Figure 2: Axial fat-suppressed T2-weighted (A), DWI $\left(b=1000 \mathrm{~s} / \mathrm{mm}^{2}\right)(B)$, unenhanced (C) and enhanced (D) fat-suppressed T1-weighted MR images show a significant stenosis (white arrow)in transverse colon that demonstrates restricted diffusion. Sagittal fat-suppressed T2-weighted (E) shows the stenosing bowel wall thickening (white arrow).
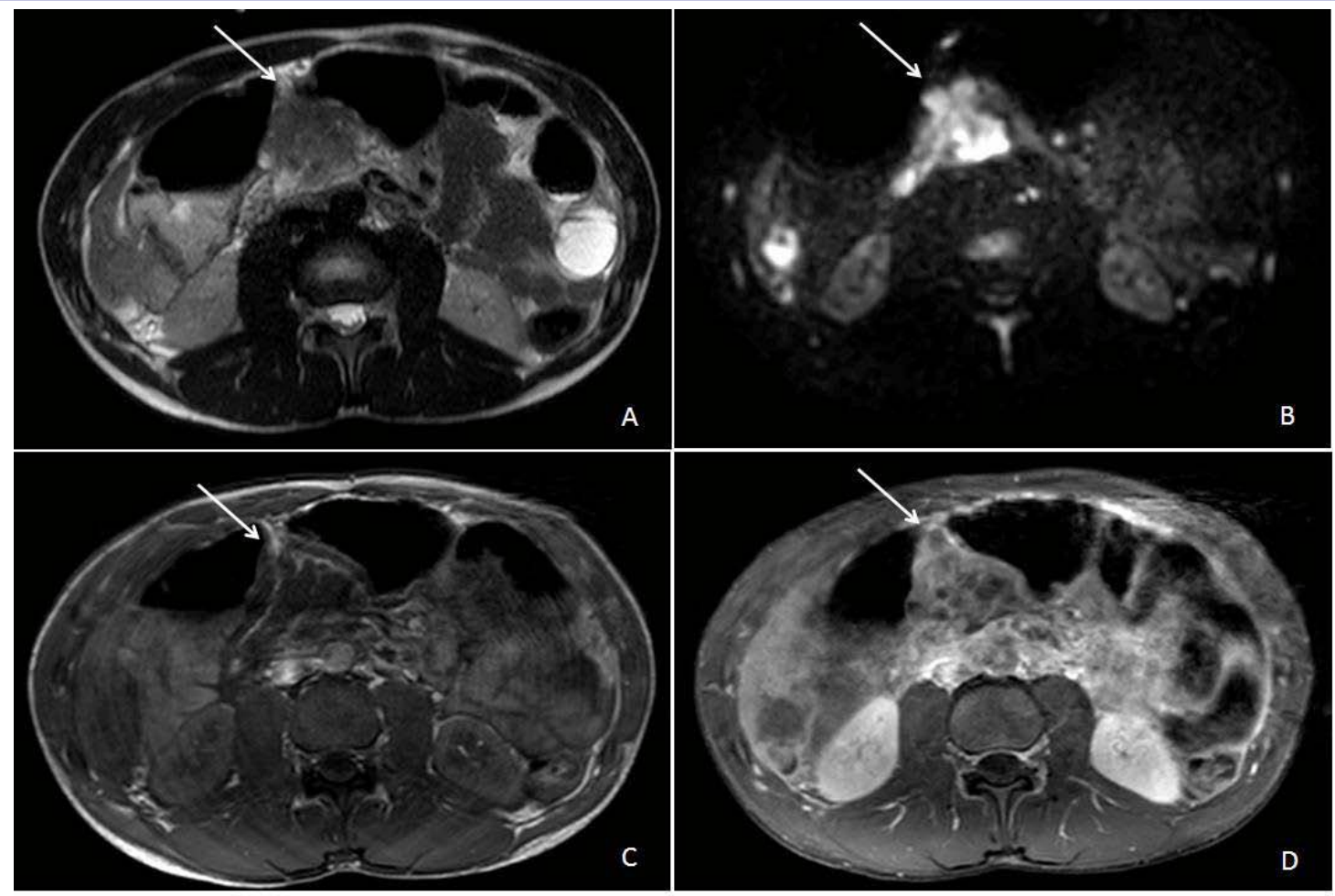

Figure 3: Axial fat-suppressed T2-weighted (A), DWI ( $b=1000 \mathrm{~s} / \mathrm{mm}^{2}$ ) (B), unenhanced (C) and enhanced (D) fat-suppressed T1-weighted MR images show the stenosing mass (white arrow) in transverse colon with restricted diffusion.

Citation: Giovanna Ognibene NM, Basile M, Schiavone R, Di Maurizio M, Defilippi C (2016) Metastatic Primary Adenocarcinoma of Colon: A Case Report of 15-Year-Old Boy. Gastroenterol Pancreatol Liver Disord 3(2): 1-8. DOI: http://dx.doi.org/10.15226/2374- 

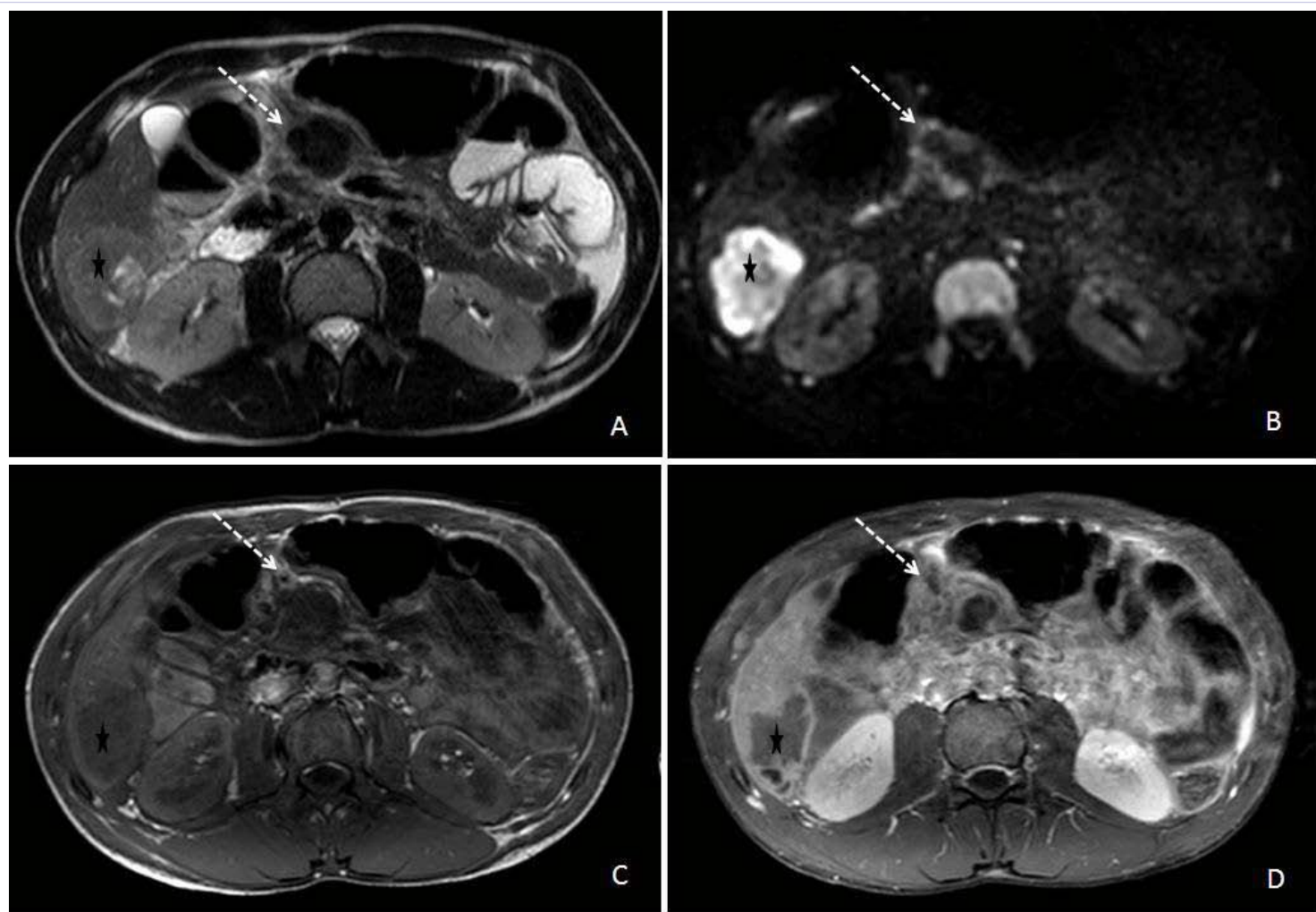

Figure 4: Axial fat-suppressed T2-weighted (A), DWI $\left(b=1000 \mathrm{~s} / \mathrm{mm}^{2}\right)(B)$, unenhanced (C) and enhanced (D) fat-suppressed T1-weighted MR images show enlarged and confluent mesenteric lymph nodes (white dotted arrow).

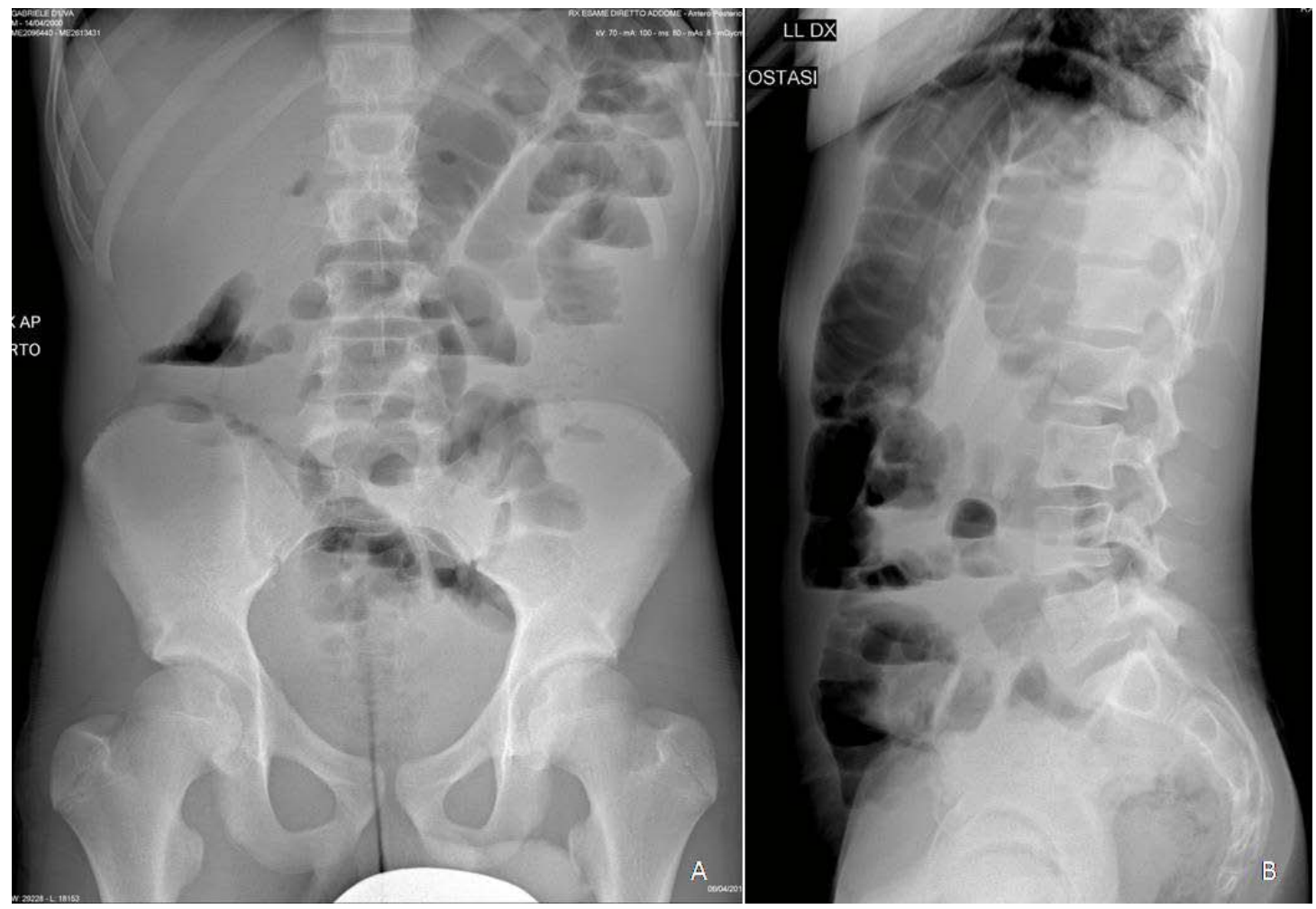

Figure 5: Abdomen X-ray (A-B) demonstrates an obstruction of the upper section of the colon with air-fluid levels.

Citation: Giovanna Ognibene NM, Basile M, Schiavone R, Di Maurizio M, Defilippi C (2016) Metastatic Primary Adenocarcinoma of Colon: A Case Report of 15-Year-Old Boy. Gastroenterol Pancreatol Liver Disord 3(2): 1-8. DOI: http://dx.doi.org/10.15226/2374- 

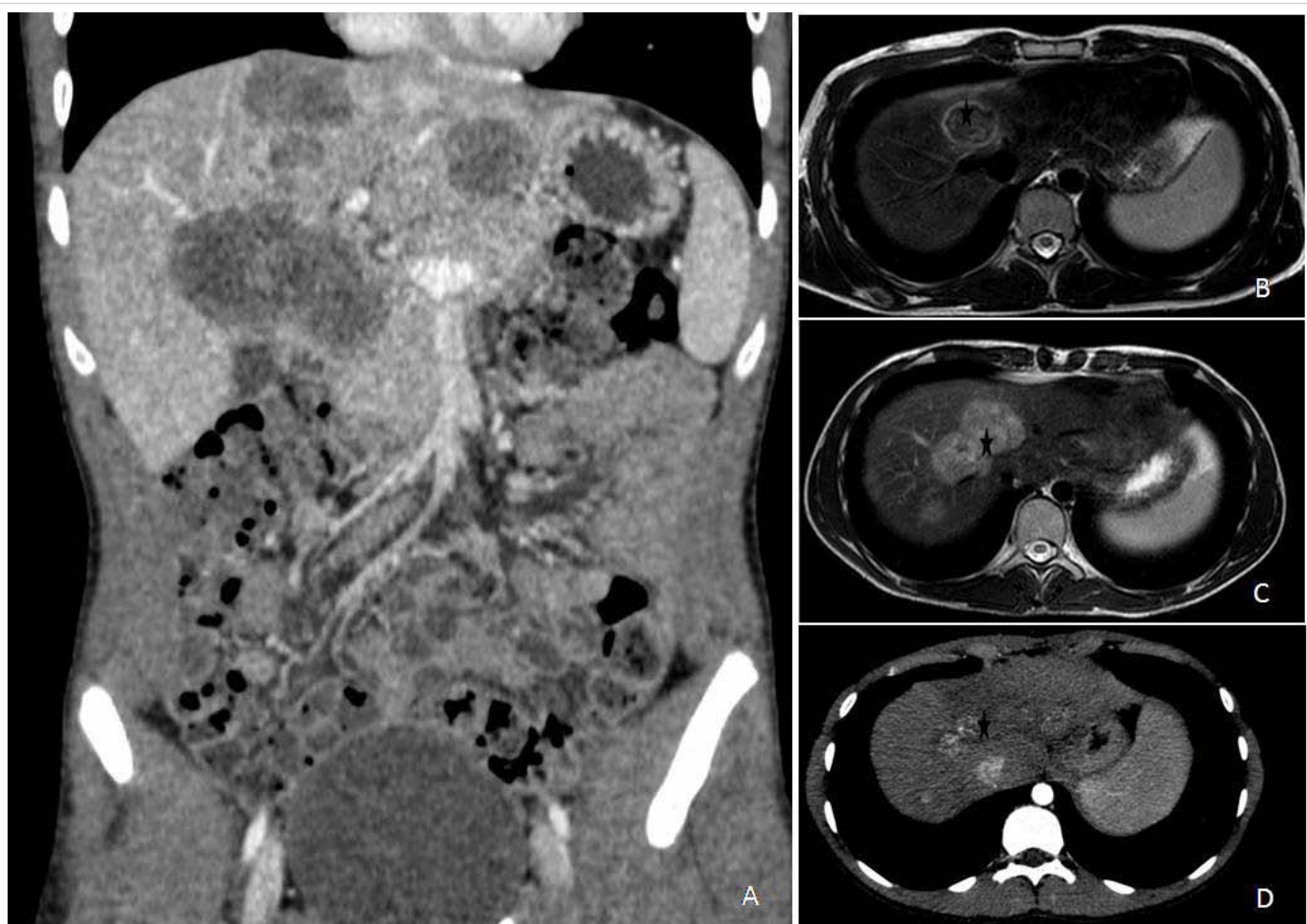

Figure 6: Coronal enhanced CT image (A) shows multiple hepatic lesions. Axial T2-weighted (B,C) MR images and axial enhanced CT images show hepatic metastasis (black star) progression during 10 months of chemo- and radio-therapy.
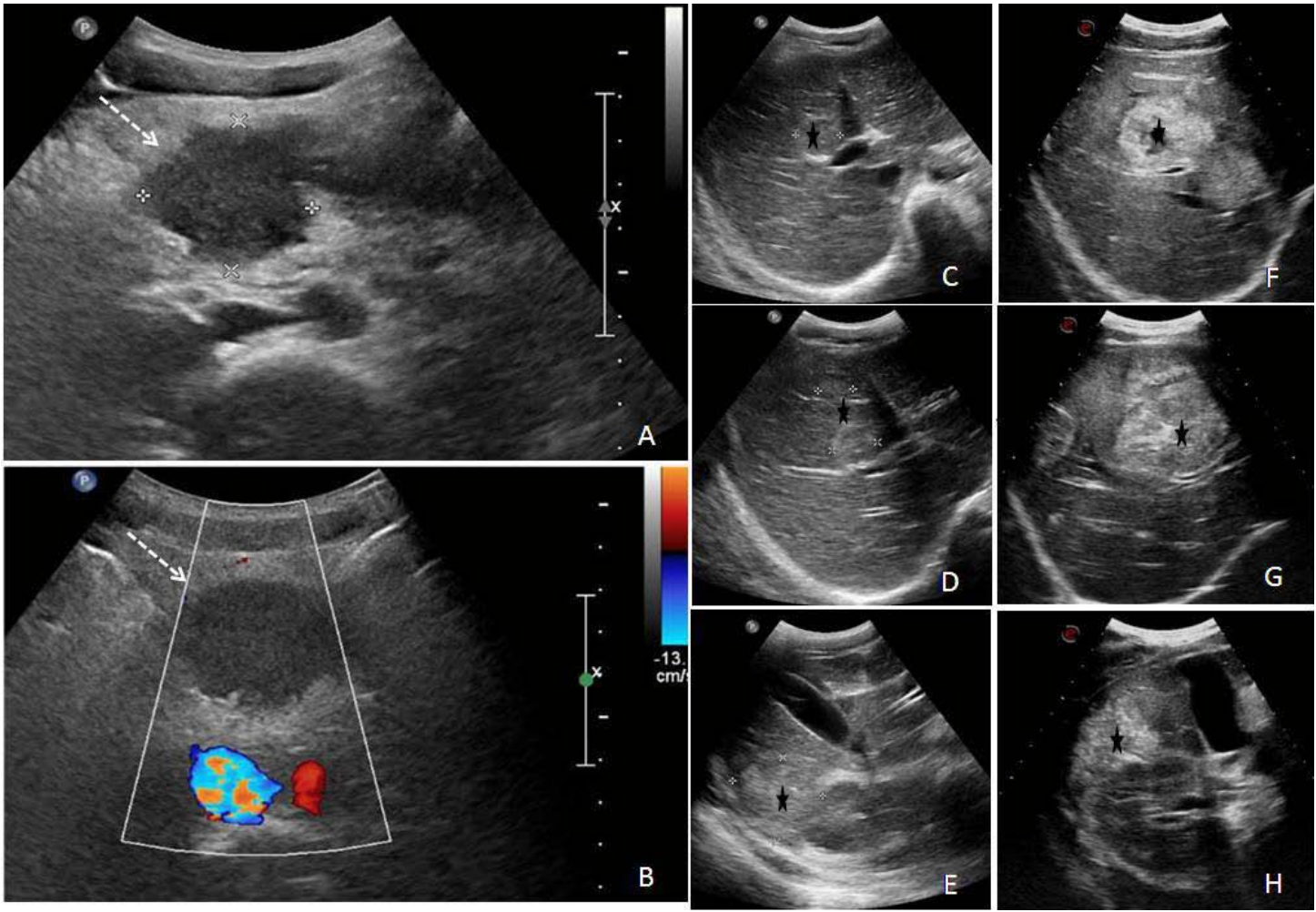

Figure 7: Ultrasonography images show: $(\mathrm{A}, \mathrm{B})$ enlarged metastatic mesenteric lymph node (white dotted arrow), the same lesion showed on figure 4; multiple liver lesions (black star) at diagnosis time (C,D,E) and after two months of chemotherapy $(\mathrm{F}, \mathrm{G}, \mathrm{H})$.

Citation: Giovanna Ognibene NM, Basile M, Schiavone R, Di Maurizio M, Defilippi C (2016) Metastatic Primary Adenocarcinoma of

Colon: A Case Report of 15-Year-Old Boy. Gastroenterol Pancreatol Liver Disord 3(2): 1-8. DOI: http://dx.doi.org/10.15226/2374-

Page 5 of 8 815X/3/2/00152 
[12] attributed the aggressive nature of this malignancy to their high rate of mucinous histology. This may be due to the fact that mucin absorbs water, swells and invades the tissue, promoting tumour growth and spread. The signet-ring subtype is associated with a much more aggressive presentation with regional lymph node involvement and diffuse peritoneal seeding [12]. Moreover, statistics also show a higher incidence of unresectable, residual disease and a higher metastasis rate in childhood CRC. Recent study by Poles et al. [26] confirms findings from prior studies demonstrating more aggressive tumor biology in children with higher proportions of signet ring and mucinous histology, less differentiation, and presentation at later stage [7-11]. This case reports a case of a moderately differentiated adenocarcinoma, uncommon on pediatric patient, with presentation as intestinal obstruction at later stage with liver and lymp nodal metastasis.

The clinical unspecific symptoms are often undervalued by parents and doctors; this therefore results in poor prognosis of advanced stage tumors, when diagnosed.

Adult cases of colorectal cancer usually present with symptoms and signs including fecal occult blood test positive (77\%), rectal bleeding (58\%), anemia (57\%), and abdominal pain (52\%) were most commonly recorded [27]. Furthermore, as high as $30 \%$ of the adult patients have advantage of early detection by screening [28]. The pediatric CRCs presenting symptoms are nonspecific and may mimic a lot of common functional gastrointestinal disorders [7]. Abdominal pain and vomiting are the most common symptoms in pediatric CRC patients [4]. In a majority of patients, the diagnosis was made only when the patient presented with symptoms of advanced intestinal obstruction, as in the current case report. Other reported symptoms include altered bowel habit, weight loss, and anemia [29]. Moreover, one must note that tumors involving the cecum and descending colon may become bulky before symptoms appear, while tumors of the rectum and sigmoid colon manifest early [21].

In young patients who develop colorectal carcinoma but have no known predisposing genetic risk factors, late diagnosis may result from the clinician's failure to consider the possibility of malignant disease in the differential diagnosis [30]. Hence, an average delay of 7.5 months from presentation of symptoms to diagnosis has been reported [18]. The possibility of a tumor should be considered for every child admitted to hospital with intestinal obstruction symptoms, incurable abdominal pain or changed bowel evacuation habits.

Once the diagnosis is suspected, contrast studies, ultrasonography and computed tomography are essential procedures for detecting the extent of the disease. However, the principal method of colorectal carcinoma diagnosis is colonoscopy, with an accuracy of 90-95\% [31] A biopsy may be obtained during colonoscopy or laparotomy, at which time definitive surgery may or may not be feasible [32]. Final diagnosis is dependent on pathological examination. CEA is a reference index of auxiliary diagnosis and relapse monitoring, though it has no tumor specificity.

Currently there are no specifically pediatric CRC treatment algorithms, so adult protocols are utilized, but in the absence of prospective trials it is unclear if this is the best option or how age affects individual treatment decisions [26]. Although, surgery is the only curative modality for localized CRCs. However, for patients with cancer staging III-IV, postoperative adjuvant chemotherapy is important to eradicate micrometastases, thereby reducing disease recurrence and increasing the cure rate [33]. At present, adjuvant chemotherapy with Oxaliplatin and 5-fluorouracil-based antineoplastic agents were among the commonly used chemotherapy combinations, as it has been found to reduce the rate of relapse and increase survival rates [3, 9]. In the modern era, monoclonal antibody target therapy such as bevacizumab also showed promising results for metastatic colorectal cancer. Postoperative radiotherapy is unable to increase the survival rate of patients with tumors that are difficult to completely resect; however, palliative radiotherapy may effectively relieve the clinical symptoms of metastasized lesions $[3,34]$.

Metastasis occurred in about $37 \%$ in young patients even during initial presentation [7]. Colon cancer metastasizes mainly to the liver, followed by the lung, peritoneum, and bone [35].

The overall survival in many series reports depends on the complete resection of the tumor and the radical resection of sentinel lymph nodes [4]. In a review of young-age colon cancer, studies comparing stage-for-stage survival found that young patients with Dukes' stage A or B tumors appear to have better survival than older patients with similar-stage disease [23]: young patients with Dukes' C or D lesions do the same or worse than older patients with the same disease stages. A large cohort study found that young patients showed poor outcome despite the similarity of their treatment to the standard therapy for adult colorectal carcinoma $[36,37]$ reflecting the preponderance of advancedstage disease in young patients. Despite standard oncologic treatment, age $\leq 21$ was a significant predictor of mortality [26]. Predictors of poor outcome aside from disease stage are incomplete resection, mucinous histology, proportion of signet-ring cells $>10 \%$, and absence of an in situ component $[7,29]$. With the advances in diagnosis and treatments, overall 5 -year survival is around $75 \%$ in adult patients but only around $51 \%$ in pediatric population [22].

The differential diagnosis includes malignant carcinoid tumor, leiomyosarcoma, malignant fibrous histiocytoma and metastatic tumor from other sites. All may have similar presentations; metastases may be identified only by histology or metastatic site [38].

\section{Conclusion}

We reported a case of a 15-year-old boy with intestinal obstruction due to a colorectal cancer without any predisposing factor. Colorectal carcinomas in children are rare and provide a lack of typical clinical symptoms and radiological data, as demonstrated in the present case study.

The present study aimed to suggest that malignancies should be considered by pediatricians and pediatric surgeons for differential diagnosis when patients present with intestinal obstruction symptoms, incurable abdominal pain or changed bowel evacuation habits without hernia or previous abdominal surgery, as early diagnosis and treatment decreases the mortality rate of patients.

\section{References}

1. Yang R, Cheung MC, Zhuge Y, Armstrong C, Koniaris LG, Sola JE. Primary solid tumors of the colon and rectum in the pediatric 
patient: A review of 270 cases. J Surg Res. 2010;161(2):209- 216. doi 10.1016/j.jss.2008.12.003.

2. Howlader N, Noone AM, Krapcho M, et al, editors. SEER Cancer Statistics Review. Bethesda, MD: National Cancer Institute; 19752011 [http://seer.cancer.gov/statfacts/ html/colorect.html, http:// seer.cancer.gov/csr/1975_2011/, based on November 2013 SEER data submission, posted to the SEER web site, April 2014

3. Yang S, Liu G, Zheng S, Dong K, Ma Y, Xiao X. Signet-ring cell carcinoma of the colon: A case report of a 9-year-old boy. Oncol Lett. 2015;10(3):1632-1634.

4. Salas-Valverde S, Lizano A, Gamboa Y, Vega S, Barrantes M Santamaría S, et al. Colon carcinoma in children and adolescents: prognostic factors and outcome-a review of 11 cases. Pediatr Surg Int 2009;25(12):1073-1076. doi:10.1007/s00383-009-2491-y

5. Wyllie R, Hyams JS, Kay M. Pediatric Gastrointestinal and Liver Disease. 4th ed. Philadelphia: Saunders; 2011.

6. Singer G, Hoellwarth ME. Colorectal carcinomas in children: an institutional experience. Pediatr Surg Int. 2012;28(6):591-595. doi:10.1007/s00383-012-3090-x.

7. Sultan I, Rodriguez-Galindo C, El-Taani H, Pastore G, Casanova M, Gallino G, et al. Distinct features of colorectal cancer in children and adolescents: a population-based study of 159 cases. Cancer. 2010; 116(3):758-765. doi: 10.1002/cncr.24777.

8. Tricoli JV, Seibel NL, Blair DG, Albritton K, Hayes-Lattin B . Unique characteristics of adolescent and young adult acute lymphoblastic leukemia, breast cancer, and colon cancer. J Natl Cancer Inst 2011;103(8):628-635. doi: 10.1093/jnci/djr094.

9. Goldberg J, Furman WL. Management of colorectal carcinoma in children and young adults. J Pediatr Hematol Oncol . 2012;34(Suppl. 2):S76-9 . doi: 10.1097/MPH.0b013e31824e38c1.

10. LaQuaglia MP, Heller G, Filippa DA, Karasakalides A, Vlamis V, Wollner $\mathrm{N}$, et al. Prognostic factors and outcome in patients 21 years and under with colorectal carcinoma. J Pediatr Surg. 1992; 27(8):1085-1089.

11. You YN, Xing Y, Feig BW, Chang GJ, Cormier JN. Young-onset colorectal cancer: is it time to pay attention? Arch Intern Med. 2012;172(3):287289. doi: 10.1001/archinternmed.2011.602.

12. Kravarusic D, Feigin E, Dlugy E, Steinberg R, Baazov A, Erez I, et al. Colorectal carcinoma in childhood: a retrospective multicenter study. J Pediatr Gastroenterol Nutr. 2007;44(2):209-211.

13. Brown RA, Rode H, Millar AJ, Sinclair-Smith C and Cywes S: Colorectal carcinoma in children. J Pediatr Surg. 1992; 27: 919-921.

14. Colangeli M, Calamelli C, Manfrini M, Frisoni T, Donati DM. Bone metastasis from colon carcinoma in an 11-year-old boy: radiological features and brief review of the literature. Skeletal Radiol. 2015;44(5):743-748. doi:10.1007/s00256-014-2039-7.

15. Karnak I, Ciftci AO, Senocak ME, Büyükpamukçu N. Colorectal carcinoma in children. J Pediatr Surg. 1999; 34(10): 1499-1504.

16. Vastyan AM, Walker J, Pintér AB, Gerrard M, Kajtar P. Colorectal carcinoma in children and adolescents - a report of seven cases. Eur J Pediatr Surg. 11(5):338-341.

17. Brown RA, Rode H, Millar AJ, Sinclair-Smith C and Cywes S: Colorectal carcinoma in children. J Pediatr Surg. 1992; 27: 919-921.

18. Bjoernsen LP, Lindsay MB. An unusual case of pediatric abdominal pain. CJEM. 2011;13(2):133-138.
19. Al-Tonbary Y, Darwish A, El-Hussein A, Fouda A. Adenocarcinoma of the colon in children: case series and mini-review of the literature. Hematol Oncol Stem CellTher. 2013;6(1):29-33. doi:10.1016/j. hemonc.2013.02.003.

20. Radhakrishnan CN, Bruce J. Colorectal cancers in children without any predisposing factors. A report of eight cases and review of the literature. Eur J Pediatr Surg. 2003;13(1):66-68.

21. Rao BN, Pratt CB, Fleming ID, Dilawari RA, Green AA, Austin BA. Colon carcinoma in children and adolescents. A review of 30 cases.Cancer. 1985;55(6):1322-1326.

22. Blumer SL, Anupindi SA, Adamson PC, Lin H, Price AP, Markowitz RI, et al. Sporadic adenocarcinoma of the colon in children: case series and review of the literature. J Pediatr Hematol Oncol. 2012;34(4):e137e141. doi: 10.1097/MPH.0b013e3182467f3e.

23. O'Connell JB, Maggard MA, Livingston EH, Yo CK. Colorectal cancer in the young. Am J Surg. 2004;187(3):343-348.

24.GLOBOCAN 2012: Estimated Cancer Incidence, Mortality and Prevalence Worldwide in 2012 [database online]. Lyon: InternationalAgency for Research on Cancer

25. Sharon E, Plon DM. Childhood cancer and heredity. In: Pizzo PA, editor. Principles and practice of pediatric oncology. LWW; 2010.

26. Poles GC, Clark DE, Mayo SW, Beierle EA, Goldfarb M, Gow KW, et al. Colorectal carcinoma in pediatric patients: A comparison with adult tumors, treatment and outcomes from the National Cancer Database. J Pediatr Surg. 2015 Dec 1. pii: S0022-3468(15)00757-5. doi: 10.1016/j. jpedsurg. 2015.11.005

27. Majumdar SR, Fletcher RH, Evans AT. How does colorectal cancer present? Symptoms, duration, and clues to, location. Am J Gastroenterol.1999;94(10):3039-3045.

28. Moiel D, Thompson J. Early detection of colon cancer-the Kaiser permanente northwest 30-year history: how do we measure success? Is it the test, the number of tests, the stage, or the percentage of screen-detected, patients? Perm J. 2011;15(4):30-38.

29. Hill DA, Furman WL, Billups CA, Riedley SE, Cain AM, Rao BN, et al. Colorectal carcinoma in childhood and adolescence: a clinicopathologic review. J Clin Oncol. 2007;25(36):5808-5814.

30. Dozois EJ, Boardman LA, Suwanthanma W, Limburg PJ, Cima RR, Bakken JL , et al. Young-onset colorec $\neg$ tal cancer in patients with no known genetic predisposition: can we in $า$ crease early recognition and improve outcome?. Medicine (Baltimore). 2008;87(5):259-263. doi: 10.1097/MD.0b013e3181881354.

31. Beck DE: Colorectal cancer screening. Clin Colorectal Surg. 200;14: 115

32. Skibber JM, Minsky BD, Hoff PM. Cancer of the colon. In: DeVita VT, Hellman S, Rosenberg SA, editors. Cancer: principles and practice of oncology. Philadelphia: Lippincott Wilkins \& Williams; 2001. p. 121671.

33. Koh KJ, Lin LH, Huang SH, Wong JU. CARE--pediatric colon adenocarcinoma: a case report and literature review comparing differences in clinical features between children and adult patients. Medicine (Baltimore). 2015;94(6):e503. doi: 10.1097/MD. 0000000000000503 .

34. Labianca R, Beretta G, Gatta G, De Braud F and Wils J: Colon cancer. Crit Rev Oncol Hematol. 2004;51: 145 -170. 
35. Attili VS, Rama Chandra C, Dadhich HK, Sahoo TP, Anupama G, et al. Unusual metastasis in colorectal cancer. Indian J Cancer. 2006;43(2):93-95.

36. Du W, Mah JT, Lee J, Sankila R, Sankaranarayanan R, Chia KS. Inci ᄀdence and survival of mucinous adenocarcinoma of the colorectum: a population-based study from an Asian country. Dis Colon Rectum. 2004;47(1):78-85.
37. Chung MY, Park YS, Ryu SR, Ahn SB, Kim SH, Jo YJ, et al. A case of colonic mucinous adenocarcinoma in 19-year-old male patient. Clin Endosc. 2012;45(1):103-107. doi: 10.5946/ce.2012.45.1.103.

38. Pappo AS, Furman WL. Management of infrequent cancers of childhood: colorectal carcinoma. In: Pizzo PA, Poplack DG, (editors). Principles and practice of pediatric oncology. Lippincott Williams \& Wilkins; 2006. p. 1174-201. 